\title{
Copeptin: a new predictor for severe obstructive sleep apnea
}

\author{
This article was published in the following Dove Press journal: \\ Therapeutics and Clinical Risk Management \\ 10 April 2015 \\ Number of times this article has been viewed
}

\author{
Halit Çınarka' \\ Servet Kayhan' \\ Mevlüt Karataș' \\ Asiye Yavuz' \\ Aziz Gümüș' \\ Songül Özyurt' \\ Medine Cumhur Cüre ${ }^{2}$ \\ Ünal Șahin' \\ 'Department of Chest Diseases, \\ ${ }^{2}$ Department of Biochemistry, Recep \\ Tayyip Erdoğan University, Rize, Turkey
}

Introduction: Copeptin which is the C-terminal fragment of antidiuretic hormone (ADH), is a biomarker that has been reported to be increased in various cardiovascular disorders, cerebrovascular diseases and associated with prognosis. Patients with obstructive sleep apnea syndrome (OSAS) have a tendency to develop coronary and cerebral atherosclerotic diseases.

Objectives: The aim of the present study was to study copeptin levels in patients with obstructive sleep apnea and in a control group in order to determine whether copeptin could be used as a biomarker predicting the severity of OSAS and possible complications in this group.

Methods: A total of 116 patients with OSAS, diagnosed by polysomnography, and 27 controls were included in the study. Blood samples were collected after overnight fasting, and copeptin levels were measured with enzyme-linked immunosorbent assay.

Results: Copeptin levels were significantly higher in the OSAS group compared to control group $(2,156 \pm 502 ; 1,845 \pm 500 \mathrm{pg} / \mathrm{mL}$, respectively, $P=0.004)$. Mean copeptin level of the patients having apnea-hypopnea index $(\mathrm{AHI}) \geq 30$ was significantly higher than that of the patients having AHI $<30(2,392 \pm 415 ; 2,017 \pm 500 \mathrm{pg} / \mathrm{mL}$, respectively, $P<0.001)$. A multivariate regression analysis showed that copeptin level, (hazard ratio: 1.58; 95\% confidence interval: 1.09-2.30) was a predictor of severe OSAS $(P=0.016)$. Copeptin levels showed significant positive correlation with AHI ( $r=0.32 ; P<0.001)$, desaturation index $(r=0.23 ; P=0.012)$, arousal index $(r=0.24$; $P=0.010)$ and $\mathrm{CRP}(r=0.26 ; P=0.011)$ respectively.

Conclusion: Copeptin levels are high in OSAS patients and copeptin is a potential marker for identifying patients with a high risk of early cardiovascular complications of OSAS. Copeptin has modest sensitivity (84\%) for discriminating severe OSAS patients who are candidates for severe cardiovascular complications.

Keywords: copeptin, obstructive sleep apnea, predictor, inflammation

\section{Introduction}

Obstructive sleep apnea syndrome (OSAS) is characterized by repetitive episodes of upper airway obstruction-related oxygen desaturation and sleep fragmentation. ${ }^{1}$ Oxygen desaturation-associated apneic episodes, negative intrathoracic pressure, arousals augmented by upper airway collapse, and repetitive episodes of activation of the sympathetic nervous system might lead to abnormal humoral, neural, metabolic, thrombotic, and inflammatory responses, and when activated, these pathways contribute to cardiovascular mortality. ${ }^{2}$ The well-known gold standard for diagnosis of OSAS is polysomnography, but polysomnography has several important limitations, including cost, limited availability, and need for cooperation on the part of the patient. In addition to helping confirm a diagnosis of OSAS, elevated biochemical or inflammatory biomarker levels might also define an increased risk of cardiovascular disease in OSAS. ${ }^{3}$

Studies have shown that copeptin is a good predictor of outcome in patients with heart failure or acute myocardial infarction, and its value has even been found
Correspondence: Servet Kayhan Department of Chest Diseases, Recep Tayyip Erdoğan University, 53200 Rize, Turkey

Tel +904642130491

Fax +904642170364

Email kayhanservet@gmail.com
Therapeutics and Clinical Risk Management 20I5:II 589-594 
to be superior to that of brain natriuretic peptide. ${ }^{4,5}$ The prognostic role of copeptin in lower respiratory tract infections and chronic obstructive pulmonary disease has also been studied, ${ }^{6,7}$ and a recent study reported that copeptin is a potential prognostic marker for short-term mortality independently and in addition to natriuretic peptide levels in patients with acute dyspnea. ${ }^{8}$ Copeptin levels may reflect both the inflammatory cytokine response and the presence of hemodynamic and osmoregulatory disturbances. ${ }^{9}$ Copeptin may also be associated with cardiovascular complications in OSAS patients. The aim of this study was to assess copeptin levels in patients with OSAS and compare them with levels in controls.

\section{Materials and methods}

\section{Patients}

The study population included 116 patients with OSAS recently diagnosed by polysomnography. Patients having a personal or family history of psychiatric illness, a history of alcoholism or substance abuse, or a central nervous system disorder were excluded.

\section{Polysomnography}

All patients underwent overnight polysomnography. All variables were recorded on a computer system (Alice Sleepware, Philips Respironics, Murrysville, PA, USA). Data were collected by electroencephalography (F3M2, F4M1, C3M2, C4M1, O1M2, O2M1), bilateral electro-oculography, submental electromyography, thoracic and abdominal movements measured by uncalibrated inductive plethysmography, oxyhemoglobin saturation, airflow through the nose and mouth recorded by thermistors, electrocardiography, snoring microphone, and video monitoring using an infrared video camera. The entire recording was done by an experienced sleep technician. Polysomnography records were scored in 30-second periods for sleep, breathing, and oxygenation. Hypopnea was defined as a reduction of $>50 \%$ in one of three respiratory signals, ie, the airflow signal, or the respiratory or abdominal signal of respiratory inductance plethysmography, with either an associated decrease of $\geq 3 \%$ in oxygen saturation or arousal. Apnea was defined as complete cessation of airflow for $\geq 10$ seconds. ${ }^{1}$ The average number of apneas and hypopneas per hour of sleep was calculated as the apneahypopnea index (AHI). Patients with an AHI $\geq 5$ events/ hour were diagnosed as having OSAS. Excessive daytime sleepiness was identified using the Epworth Sleepiness Scale, and values over 10 were accepted as a pathological result. ${ }^{10}$ Arousals were scored according to accepted definitions. ${ }^{11}$

\section{Laboratory analysis}

Serum copeptin levels were quantified by enzyme-linked immunosorbent assay using commercially available matched antibodies (USCN Life Science İnc., Wuhan, People's Republic of China). The intra-assay and interassay coefficients of variation were $<10.0 \%$ and $<12.0 \%$, respectively. The sensitivity was calculated to be $6.1 \mathrm{pg} / \mathrm{mL}$.

\section{Statistical analysis}

The Kolmogorov-Smirnov test was used to test for a normal distribution of continuous variables. Data characterized by a normal distribution were expressed as the mean \pm standard deviation. Parameters without such a distribution were expressed as the median with range. The Student's $t$-test (normal distribution) or the Mann-Whitney $U$-test (non-normal distribution) was used for comparing the two groups. Analysis of variance (normal distribution) or the Kruskal-Wallis test (non-normal distribution) was used to compare more than two groups. Discrete variables were compared using Fisher's Exact test. The Spearman test was used to assess the correlation between variables. Multivariate regression analysis was used as a stepwise descending method from prognostic factors, with a significance of $<0.1$ in univariate analysis. Stepwise multivariate linear regression was also performed to determine the factors independently associated with cardiovascular disease. A criterion of $P<0.05$ for entry and $P<0.10$ for removal was imposed in this procedure. $P<0.05$ was considered to be statistically significant.

\section{Results}

\section{Clinical baseline characteristics}

A total of 116 patients and 27 controls were included in the study population. The clinical characteristics of the OSAS and control groups are shown in Table 1. Copeptin levels were significantly higher in the OSAS group than in the control group $(2,156 \pm 502 \mathrm{pg} / \mathrm{mL}$ and $1,845 \pm 500 \mathrm{pg} / \mathrm{mL}$, respectively, $P=0.004$, Figure 1$)$.

Table I Clinical characteristics of the study group

\begin{tabular}{lll}
\hline Characteristics & $\begin{array}{l}\text { OSAS group } \\
\text { (mean } \pm \text { SD) }\end{array}$ & $\begin{array}{l}\text { Control group } \\
\text { (mean } \pm \text { SD) }\end{array}$ \\
\hline Age, years & $48 \pm 1.04$ & $43 \pm 1.25$ \\
$\mathrm{BMI}$ & $33 \pm 6$ & $3 \mathrm{I} \pm 6$ \\
$\mathrm{AHI}$ & $30 \pm 2.43$ & $3 \pm 3.04$ \\
Copeptin & $2,156 \pm 502$ & $\mathrm{I}, 845 \pm 500$ \\
\hline
\end{tabular}

Abbreviations: AHI, apnea-hypopnea index; BMI, body mass index; OSAS, obstructive sleep apnea syndrome. 


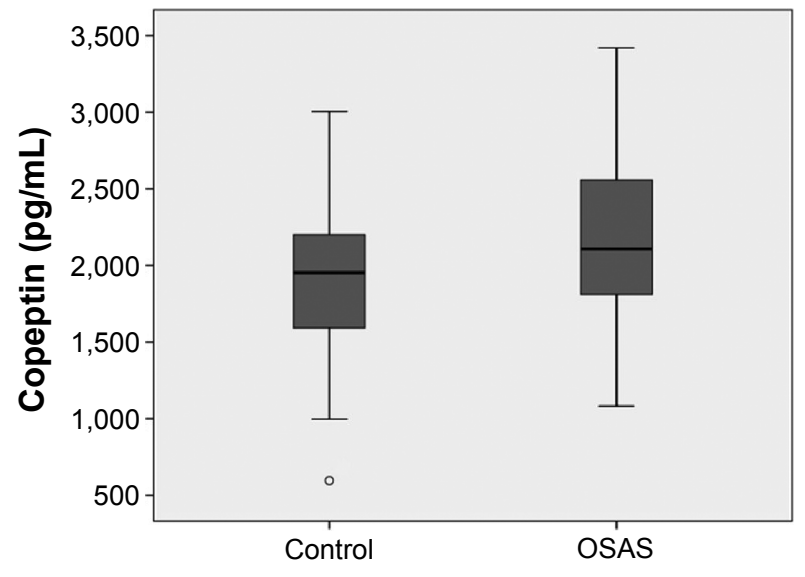

Figure I Comparison of copeptin levels between patients with obstructive sleep apnea and healthy control group.

Abbreviation: OSAS, obstructive sleep apnea syndrome.

\section{Association of copeptin levels with OSAS severity}

Copeptin levels in patients with $\mathrm{AHI} \geq 30$ were significantly higher than those in patients with $\mathrm{AHI}<30(2,392 \pm 415$ and $2,017 \pm 500 \mathrm{pg} / \mathrm{mL}$, respectively, $P<0.001$, Figure 2 ). The optimal cut-off copeptin value for predicting severe OSAS was $2,044 \mathrm{pg} / \mathrm{mL}$. The area under the curve of copeptin for prediction of severe OSAS was 0.736 ( $95 \%$ confidence interval [CI] 0.647-0.825, $P<0.001$, Figure 3 ). In cases with copeptin levels $>2,044 \mathrm{pg} / \mathrm{mL}$, the specificity and sensitivity for severe OSAS were 58\% (95\% CI $0.45-0.69)$ and $84 \%$ (95\% CI 0.69-0.93), respectively. The negative predictive value of copeptin at a cut-off of $2,044 \mathrm{pg} / \mathrm{mL}$ for predicting severe OSAS was $86 \%$ (95\% CI $0.72-0.94)$ and the positive predictive value of copeptin at a cut-off of $2,044 \mathrm{pg} / \mathrm{mL}$ for severe OSAS was $54 \%$ (95\% CI $0.41-0.66)$.

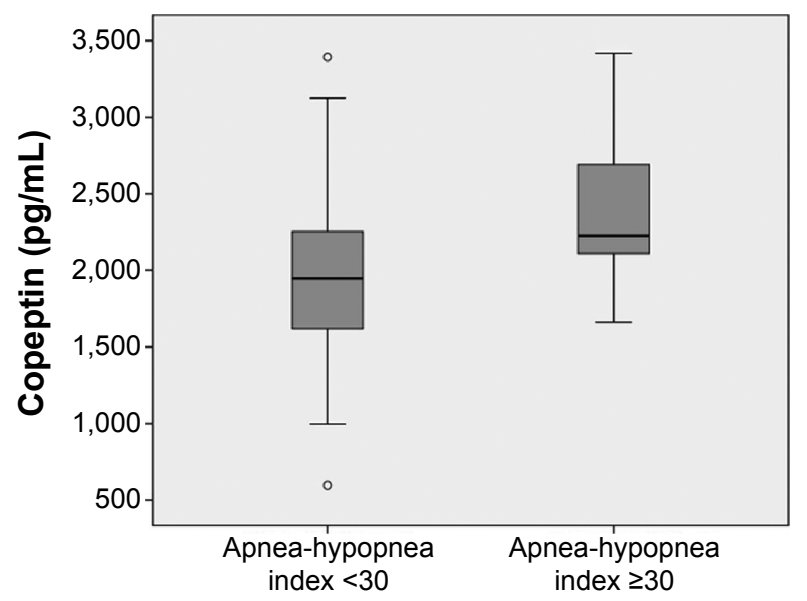

Figure 2 Comparison of copeptin levels between the patients with apnea-hypopnea index $(\mathrm{AHI}) \geq 30$ and the patients with $\mathrm{AHI}<30$.

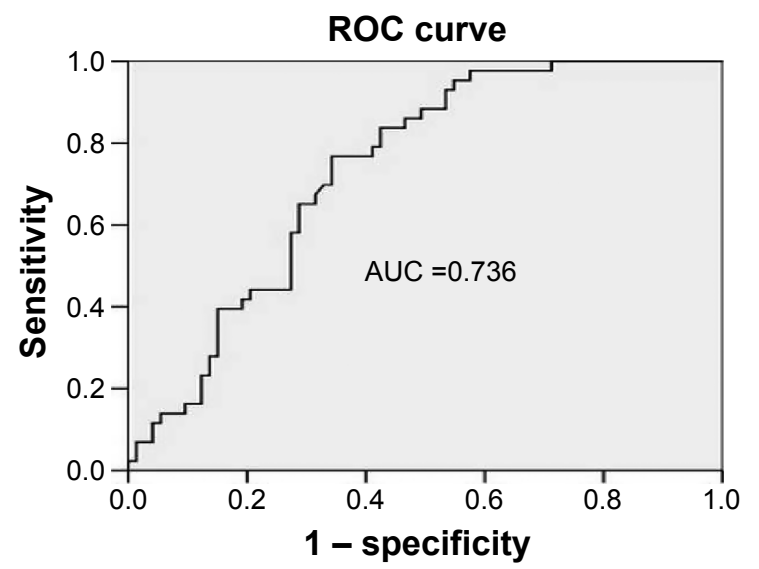

Figure 3 Receiver operating characteristic curve analysis of copeptin levels in predicting severe OSAS (the area under the curve of copeptin for prediction of severe OSAS was 0.736 with the optimal cut-off copeptin level of $2,044 \mathrm{pg} / \mathrm{mL}$; the specificity and sensitivity for severe OSAS were found as $58 \%$ and $84 \%$ respectively).

Abbreviations: OSAS, obstructive sleep apnea syndrome; ROC, receiver operating characteristic; AUC, area under the curve.

\section{Association and correlation of copeptin levels with other clinical characteristics}

In univariate analysis, a significant association was found between severe OSAS and age, body mass index, copeptin level, and C-reactive protein (CRP) level. CRP and copeptin levels were analyzed from the same blood samples of the study population. A multivariate regression analysis showed that the copeptin level (hazard ratio $1.58,95 \%$ CI 1.09-2.30) was a predictor of severe OSAS $(P=0.016)$. Other potential predictors for severe OSAS on multivariate regression analysis are summarized in Table 2. Copeptin levels showed a significant positive correlation with AHI $(r=0.32 ; P<0.001$, Figure 4), desaturation index ( $r=0.23 ; P=0.012$, Figure 5), arousal index ( $r=0.24 ; P=0.010$, Figure 6$)$, and CRP level $(r=0.26 ; P=0.011$, Figure 7$)$.

\section{Discussion}

In the present study, we found an increased level of copeptin in patients with OSAS when compared with healthy controls. Multivariable logistic regression analysis revealed that copeptin levels were an independent predictor of severity of

Table 2 Independent predictors of severe OSAS in the study population

\begin{tabular}{llll}
\hline Variables & OR & $\mathbf{9 5 \%} \mathbf{C l}$ & $P$-value \\
\hline Age & $1.03 \mathrm{I}$ & $0.98 \mathrm{I}-\mathrm{I} .083$ & $0.23 \mathrm{I}$ \\
Body mass index & 1.065 & $0.983-\mathrm{I} .153$ & $0.12 \mathrm{I}$ \\
C-reactive protein & 1.28 & $0.528-3.105$ & 0.584 \\
Copeptin levels $>2,044 \mathrm{pg} / \mathrm{mL}$ & 6.84 & $2.212-2 \mathrm{I} .162$ & $0.00 \mathrm{I}$ \\
\hline
\end{tabular}

Abbreviations: $\mathrm{Cl}$, confidence interval; OR, odds ratio; OSAS, obstructive sleep apnea syndrome. 


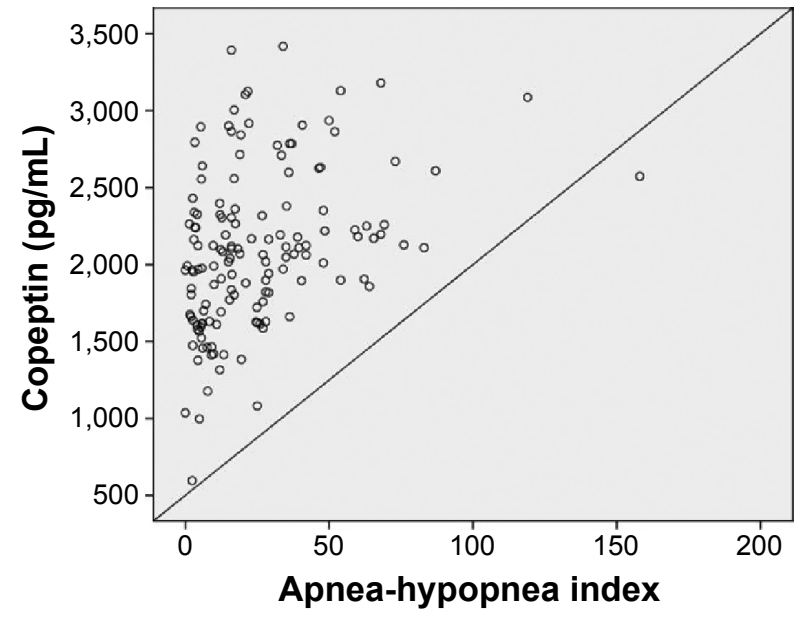

Figure 4 Correlation analysis by Spearman test between copeptin levels and apneahypopnea index $(r=0.32 ; P<0.00 I)$.

OSAS. Copeptin levels had a significant positive correlation with AHI, desaturation index, arousal index, and CRP level. Inflammation has been suggested as a potential mechanism for OSAS. High levels of various biomarkers of inflammation, such as tumor necrosis factor-alpha, interleukin-6, and CRP, have been proposed to be associated with OSAS. ${ }^{12,13}$ Copeptin has been reported to be elevated in coronary heart disease and central nervous system disorders, including acute ischemic stroke and subarachnoid hemorrhage caused by aneurysm. ${ }^{14-16}$ Higher copeptin levels are reported to be related to a poor outcome and to poor prognostic factors in patients with coronary heart disease or chronic stable heart failure. ${ }^{14,17}$ It is known that there is an increased risk of ischemic heart disease and neurological complications in patients with OSAS. ${ }^{18,19}$

We found that copeptin levels were significantly higher in OSAS patients than in controls, and this could

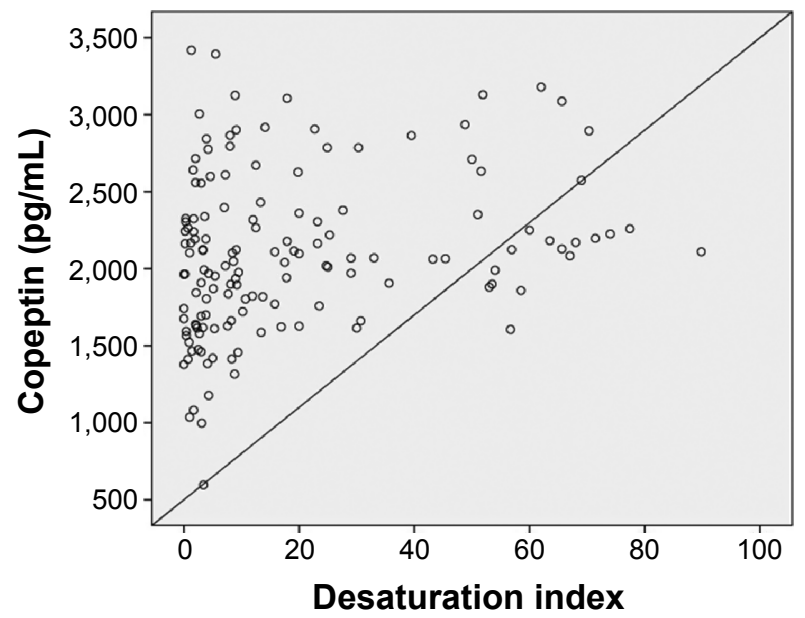

Figure 5 Correlation analysis by Spearman test between copeptin levels and desaturation index $(r=0.23 ; P=0.012)$.

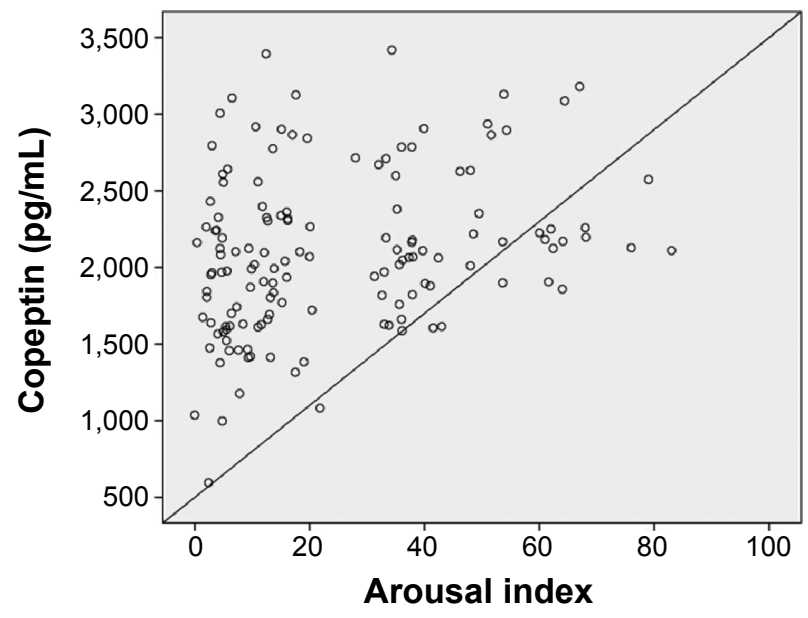

Figure 6 Correlation analysis by the Spearman test between copeptin levels and arousal index $(r=0.24 ; P=0.010)$.

be particularly important for prediction of the cardiac and neurological complications of OSAS. Intermittent obstruction of the upper airway and obstruction-induced hypoxia seen in OSAS patients is believed to increase cardiovascular risk by increasing sympathetic activation and oxidative stress. Oxidative stress and increased sympathetic activation, namely endogenous stress, lead to release of antidiuretic hormone. ${ }^{14,20,21}$ Recurrent episodes of upper airway obstruction followed by adequate ventilation lead to multiple hypoxia and reoxygenation periods. These repetitive episodes are proposed to play a role in hypoxiareperfusion injury, which augments endogenous oxidative stress. ${ }^{22}$ Release of antidiuretic hormone, and its more stable metabolite copeptin, may be expected to increase in patients with OSAS and this endogenous stress. Given that patients with OSAS already have an increased risk of cardiovascular

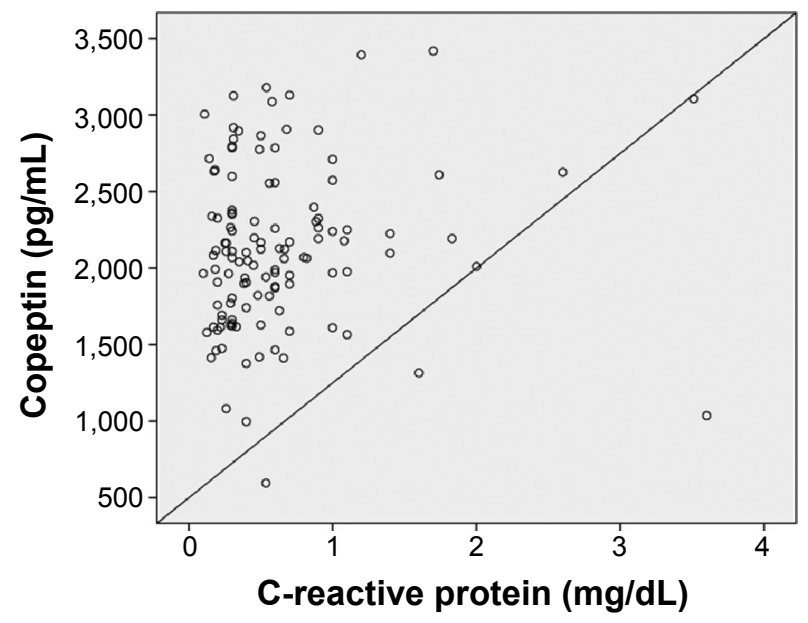

Figure 7 Correlation analysis by the Spearman test between copeptin levels and C-reactive protein level $(r=0.26 ; P=0.011)$. 
disease, copeptin might be a useful biomarker to define the cardiovascular risk level in patients who have not shown manifestations of cardiovascular disease.

In the present study, we found that copeptin levels were significantly higher in patients with an $\mathrm{AHI} \geq 30$ than in those with an $\mathrm{AHI}<30$. We also found a significant positive correlation between copeptin levels, AHI, desaturation index, and arousal index. The risk of fatal and nonfatal cardiovascular complications is 2.8 and 3.5 higher, respectively, in OSAS patients having an AHI $>30$ when compared with the healthy population. ${ }^{23}$ Therefore, patients with severe OSAS are likely to have serious cardiovascular complications. Copeptin was found as an independent predictor of severe OSAS in this study, and high levels of copeptin may also be a useful biomarker of cardiovascular disease in patients with OSAS. Copeptin may be used to identify a need for early continuous positive airway pressure (CPAP) treatment and/or to evaluate the response to CPAP in patients with OSAS. From the point of view of oxidative stress and increased sympathetic activation, which leads to increased antidiuretic hormone and ultimately increased copeptin levels, treatment with CPAP may decrease copeptin levels in the subgroup with an AHI $>30$. However, this approach needs to be addressed in further studies.

Copeptin levels also showed a significant positive correlation $(r=0.26 ; P=0.011)$ with $\mathrm{CRP}$, which is associated with inflammation, in our OSAS patients. Inflammation has an important role in the pathophysiology of OSAS, particularly in the presence of cardiovascular disease. ${ }^{24}$ Inflammatory biomarkers, including CRP, interleukin-6, and tumor necrosis factor-alpha, increase with OSAS..$^{25-27}$ Levels of CRP and interleukin-6 and spontaneous production of interleukin- 6 by monocytes are also decreased by CPAP in OSAS patients. ${ }^{12}$

There is only one study in the literature that found lower copeptin levels in patients with OSAS, ${ }^{28}$ although there is an increased risk for neurological and cardiac diseases in OSAS patients and copeptin has been found elevated in these diseases. ${ }^{14-16}$ In this study, receiver operating characteristic curve analysis was used to determine sensitivity and specificity of copeptin levels for the diagnosis of OSAS, with an area under the curve of 0.71 (95\% CI 0.60-0.82, $P<0.01$ ) for a cut-off value below $0.5 \mathrm{ng} / \mathrm{mL}$, copeptin had $67 \%$ sensitivity and $69 \%$ specificity for diagnosing OSAS. ${ }^{28}$ Another biomarker known as omentin-1 has also been found to be significantly decreased in OSAS patients compared with healthy control controls. In one study, multivariate logistic regression analysis showed that serum omentin-1 levels were inversely associated with the presence of OSAS. ${ }^{29}$ Other potential biomarkers identified in OSAS patients include P-selectin, adiponectin, leptin, and cystatin-C. . $30-32^{3}$

In the present study, it was found that the optimal cut-off value for copeptin in predicting severe OSAS was $2,044 \mathrm{pg} / \mathrm{mL}$. The area under the curve for copeptin in the prediction of severe OSAS was 0.736 (95\% CI 0.647-0.825, $P<0.001)$ with copeptin levels $>2,044 \mathrm{pg} / \mathrm{mL}$, and the specificity and sensitivity for severe OSAS were 58\% (95\% CI $0.45-0.69$ ) and $84 \%$ (95\% CI 0.69-0.93), respectively. Therefore, copeptin may be used to identify patients with severe OSAS, and may be particularly important for prediction of future cardiovascular complications.

The main limitation of our study is its small control group. Although the study included an acceptable number of OSAS patients from the point of view of power of the study, it could be considered that the number of controls was too small. A second limitation could be the lack of investigation for a circadian rhythm in copeptin levels. However, in contrast with cortisol, copeptin levels have not demonstrated any consistent circadian rhythm. ${ }^{33}$

\section{Conclusion}

Copeptin is a potential candidate marker for identifying patients at high risk of early cardiovascular complications from OSAS. However, the benefit of such biomarker-driven strategies still has to be confirmed by large randomized controlled trials. Increased copeptin levels could be related to the prognosis of OSAS. Copeptin has modest sensitivity (84\%) for discriminating patients with severe OSAS who are candidates for severe cardiovascular complications. Therefore, use of copeptin as a biomarker to predict the severity of OSAS and its complications, particularly cardiovascular events, may be recommended after further studies addressing the role of copeptin as a biomarker in patients with OSAS.

\section{Acknowledgment}

This research was supported by a grant from Recep Tayyip Erdoğan University.

\section{Disclosure}

The authors report no conflicts of interest in this work.

\section{References}

1. [No authors listed]. Sleep-related breathing disorders in adults: recommendations for syndrome definition and measurement techniques in clinical research. Report of an American Academy of Sleep Medicine Task Force. Sleep. 1999;22:667-689.

2. Shamsuzzaman AS, Gersh BJ, Somers VK. Obstructive sleep apnea: implications for cardiac and vascular disease. JAMA. 2003;290 1906-1914. 
3. De Luca Canto G, Pacheco-Pereira C, Aydinoz S, Major PW, FloresMir C, Gozal D. Biomarkers associated with obstructive sleep apnea: a scoping review. Sleep Med Rev. 2014;23C:28-45.

4. Voors AA, von Haehling S, Anker SD, et al. C-terminal provasopressin (copeptin) is a strong prognostic marker in patients with heart failure after an acute myocardial infarction: results from the OPTIMAAL Study. Eur Heart J. 2009;30:1187-1194.

5. Khan SQ, Dhillon OS, O'Brien RJ, et al. C-terminal provasopressin (copeptin) as a novel and prognostic marker in acute myocardial infarction: Leicester Acute Myocardial Infarction Peptide (LAMP) study. Circulation. 2007;115:2103-2110.

6. Müller B, Morgenthaler N, Stolz D, et al. Circulating levels of copeptin, a novel biomarker, in lower respiratory tract infections. Eur J Clin Invest. 2007;37:145-152.

7. Stolz D, Christ-Crain M, Morgenthaler NG, et al. Copeptin, C-reactive protein, and procalcitonin as prognostic biomarkers in acute exacerbation of COPD. Chest. 2007;131:1058-1067.

8. Potocki M, Breidthardt T, Mueller A, et al. Copeptin and risk stratification in patients with acute dyspnea. Crit Care. 2010;14:213-221.

9. Lin Q, Fu F, Chen H, Zhu B. Copeptin in the assessment of acute lung injury and cardiogenic pulmonary edema. Respir Med. 2012;106: 1268-1277.

10. Johns MW. A new method for measuring daytime sleepiness: the Epworth Sleepiness Scale. Sleep. 1991;14:540-545.

11. [No authors listed]. EEG arousals: Scoring rules and examples: a preliminary report from the sleep disorders ATLAS Task Force of the American Sleep Disorders Association. Sleep. 1992;15:173-184.

12. Yokoe T, Minoguchi K, Matsuo H, et al. Elevated levels of C-reactive protein and interleukin- 6 in patients with obstructive sleep apnea syndrome are decreased by nasal continuous positive airway pressure. Circulation. 2003;107:1129-1134.

13. Calvin AD, Albuquerque FN, Lopez-Jimenez F, Somers VK. Obstructive sleep apnea, inflammation, and the metabolic syndrome. Metab Syndr Relat Disord. 2009;7:271-278.

14. Potocki M, Reichlin T, Thalmann S, et al. Diagnostic and prognostic impact of copeptin and high-sensitivity cardiac troponin $\mathrm{T}$ in patients with pre-existing coronary artery disease and suspected acute myocardial infarction. Heart. 2010;98:558-565.

15. Katan M, Fluri F, Morgenthaler NG, et al. Copeptin: a novel, independent prognostic marker in patients with ischemic stroke. Ann Neurol. 2009;66:799-808.

16. Zhu XD, Chen JS, Zhou F, Liu QC, Chen G, Zhang JM. Detection of copeptin in peripheral blood of patients with aneurysmal subarachnoid hemorrhage. Crit Care. 2011;15:R288.

17. Tentzeris I, Jarai R, Farhan S, et al. Complementary role of copeptin and high-sensitivity troponin in predicting outcome in patients with stable chronic heart failure. Eur J Heart Fail. 2011;13: 726-733.
18. Cifçi N, Uyar M, Elbek O, Süyür H, Ekinci E. Impact of CPAP treatment on cardiac biomarkers and pro-BNP in obstructive sleep apnea syndrome. Sleep Breath. 2010;14:241-244.

19. Oktay B, Akbal E, Firat H, Ardic S, Akdemir R, Kizilgun M. Evaluation of the relationship between heart type fatty acid binding protein levels and the risk of cardiac damage in patients with obstructive sleep apnea syndrome. Sleep Breath. 2008;12:223-228.

20. Morgenthaler NG, Struck J, Alonso C, Bergmann A. Assay for the measurement of copeptin, a stable peptide derived from the precursor of vasopressin. Clin Chem. 2006;52:112-119.

21. Enhörning S, Wang TJ, Nilsson PM, et al. Plasma copeptin and the risk of diabetes mellitus. Circulation. 2010;121:2102-2108.

22. Archontogeorgis K, Nena E, Papanas N, Steiropoulos P. Biomarkers to improve diagnosis and monitoring of obstructive sleep apnea syndrome: current status and future perspectives. Pulm Med. 2014;2014:930535.

23. Marin JM, Carrizo SJ, Vicente E, Agusti AG. Long-term cardiovascular outcomes in men with obstructive sleep apnoea-hypopnoea with or without treatment with continuous positive airway pressure: an observational study. Lancet. 2005;365:1046-1053.

24. Ryan S, Taylor CT, McNicholas WT. Systemic inflammation: a key factor in the pathogenesis of cardiovascular complications in obstructive sleep apnoea syndrome? Thorax. 2009;64:631-636.

25. Lui MM, Lam JC, Mak HK, et al. C-reactive protein is associated with obstructive sleep apnea independent of visceral obesity. Chest. 2009;135:950-956.

26. Minoguchi K, Tazaki T, Yokoe T, et al. Elevated production of tumor necrosis factor-alpha by monocytes in patients with obstructive sleep apnea syndrome. Chest. 2004;126:1473-1479.

27. Mehra R, Storfer-Isser A, Kirchner HL, et al. Soluble interleukin 6 receptor: a novel marker of moderate to severe sleep-related breathing disorder. Arch Intern Med. 2006;166:1725-1731.

28. Ozben S, Guvenc TS, Huseyinoglu N, et al. Low serum copeptin levels in patients with obstructive sleep apnea. Sleep Breath. 2013;17:1187-1192.

29. Wang Q, Feng X, Zhou C, Li P, Kang J. Decreased levels of serum omentin-1 in patients with obstructive sleep apnoea syndrome. Ann Clin Biochem. 2013;50:230-235.

30. Cofta S, Wysocka E, Dziegielewska-Gesiak S, et al. Plasma selectins in patients with obstructive sleep apnea. Adv Exp Med Biol. 2013;756: 113-119.

31. Tauman R, Serpero LD, Capdevila OS, et al. Adipokines in children with sleep disordered breathing. Sleep. 2007;30:443-449.

32. Zhang XB, Lin QC, Deng CS, Chen GP, Cai ZM, Chen H. Elevated serum cystatin $\mathrm{C}$ in severe OSA younger men without complications. Sleep Breath. 2013;17:235-241.

33. Darzy KH, Dixit KC, Shalet SM, Morgenthaler NG, Brabant G. Circadian secretion pattern of copeptin, the C-terminal vasopressin precursor fragment. Clin Chem. 2010;56:1190-1191.
Therapeutics and Clinical Risk Management

\section{Publish your work in this journal}

Therapeutics and Clinical Risk Management is an international, peerreviewed journal of clinical therapeutics and risk management, focusing on concise rapid reporting of clinical studies in all therapeutic areas, outcomes, safety, and programs for the effective, safe, and sustained use of medicines. This journal is indexed on PubMed Central, CAS,

\section{Dovepress}

EMBase, Scopus and the Elsevier Bibliographic databases. The manuscript management system is completely online and includes a very quick and fair peer-review system, which is all easy to use. Visit http://www.dovepress.com/testimonials.php to read real quotes from published authors. 